\title{
Resultados Imediatos e Tardios do Implante de Stents Farmacológicos para o Tratamento de Bifurcações: Comparação entre as Estratégias de Um Stent Versus Dois Stents
}

Breno Oliveira Almeida ${ }^{1}$, Marco Aurélio Magalhães ${ }^{1}$, Fábio Sândoli de Brito Jr. ${ }^{1}$, Alexandre Abizaid ${ }^{1}$, Tereza C. Nascimento ${ }^{1}$, Ivanise Gomes ${ }^{1}$, Marco A. Perin ${ }^{1}$

\section{RESUMO}

Fundamentos: Os stents farmacológicos reduziram a necessidade de nova revascularização nas lesões bifurcadas. Entretanto, o seguimento tardio e a estratégia mais adequada (um stent vs. dois stents) não estão bem estabelecidos. Método: No período de junho de 2002 a dezembro de 2006, 156 pacientes consecutivos com lesões envolvendo bifurcações foram tratados com implante de stents farmacológicos. Comparou-se a taxa de eventos cardíacos adversos maiores (ECAM) combinados - óbito, infarto e revascularização do vaso-alvo (RVA) - e a incidência de trombose do stent conforme a estratégia (um stent: ramo principal vs. dois stents: ramos principal e lateral). Resultados: A média das idades foi de $64 \pm 12$ anos, $75,6 \%$ eram do sexo masculino e $28,8 \%$, diabéticos. O seguimento médio foi de $2,4 \pm 1,3$ anos. Utilizou-se a estratégia com um stent em $67,3 \%$ dos pacientes. Bifurcações verdadeiras ocorreram em $64,7 \%$ dos casos. A estratégia de dois stents foi adotada em $32,7 \%$ dos casos (crush: 14,7\%; cullote: 3,2\%; T-stent: 10,3\%; simultaneous kissing stent: $3,8 \%$; V-stent: $0,6 \%$ ). Kissing balloon final foi utilizado em $85,9 \%$ dos casos. A sobrevida livre de ECAM estimada foi de $95 \%$ na estratégia com um stent vs. $86 \%$ com dois stents (teste do log-rank, p =0,03). A análise multivariada identificou o uso de dois stents como único preditor independente de ECAM (OR: 2,82; IC 95\%: 1,04$7,68 ; p=0,04)$. A taxa de trombose no emprego de um stent em comparação com dois stents foi de $1,9 \%$ vs. $3,9 \%$ $(p=0,6)$. Conclusões: $O$ emprego dos stents farmacológicos nas bifurcações apresenta baixas taxas de eventos combinados no seguimento tardio. O uso de dois stents associou-se

\section{SUMMARY}

\section{Immediate and Late Results of Drug-Eluting Stent Implantation for the Treatment of Coronary Bifurcation Lesions: Comparison Between One-Stent and Two-Stent Strategies}

Background: Drug-eluting stents (DES) have reduced the need for repeat revascularization in coronary bifurcation lesions. However, late follow-up and the most adequate strategy (one stent $v s$. two stents) have not been well established. Methods: From June 2002 to December 2006, 156 consecutive patients underwent drug-eluting stent implantation to treat coronary bifurcation lesions. The rates of major adverse cardiac events (MACE) - death, myocardial infarction, and target-vessel revascularization (TVR) - and stent thrombosis were compared according to the strategy adopted (one-stent: main branch vs. twostents: main branch and side branch). Results: The mean age of the group was $64 \pm 12$ years; $75.6 \%$ of the patients were men and $28.8 \%$ were diabetic. The mean follow-up duration was $2.4 \pm 1.3$ years. The one-stent strategy was used in $67.3 \%$ of the patients. $64.7 \%$ of the lesions treated were true bifurcations. The two-stent techniques strategy were used in $32.7 \%$ of the cases (crush: $14.7 \%$; cullote: 3.2\%; T-stenting: 10.3\%; simultaneous kissing stent: $3.8 \%$; V-stenting: $0.6 \%$ ). Final kissing balloon inflation was used in $85.9 \%$ of the cases. The combined event-free survival was estimated to be $95 \%$ with the one-stent strategy vs. $86 \%$ with the two-stent strategy ( $p$ Log Rank $=0.03$ ). Multivariate analysis identified the two-stent strategy as the only independent predictor of MACE (OR: 2.82; IC 95\%:
1 Hospital Israelita Albert Einstein - São Paulo, SP.

Correspondência: Breno Oliveira Almeida. Rua Domingos Lopes da Silva, 650/112 - Vila Suzana - São Paulo, SP. CEP 05641-030

E-mail: boalmeida@uol.com.br

Recebido em: 13/6/2008 • Aceito em: 24/10/2008 
a maior taxa de ECAM, embora constitua um subgrupo de maior complexidade.

DESCRITORES: Stents farmacológicos. Angioplastia transluminal percutânea coronária/métodos. Resultado de tratamento. Estenose coronária/terapia.

0 tratamento das lesões coronarianas em bifurcações representa um dos mais desafiadores subgrupos da intervenção percutânea. Está associado a menores taxas de sucesso imediato e a maior incidência de eventos cardíacos adversos maiores (ECAM) e reestenose se comparado ao tratamento de lesões que não envolvem bifurcações ${ }^{1}$.

A experiência acumulada no tratamento desse tipo de lesão com stents convencionais sugere que, quando possível, o emprego de estratégias complexas deve ser evitado. O implante sistemático de stents convencionais em ambos os ramos foi incapaz de reduzir eventos adversos se comparado à estratégia mais simples, com apenas um stent ${ }^{2,3}$. Com a introdução dos stents farmacológicos e a demonstração sistemática da redução das taxas de revascularização do vaso-alvo (RVA), ressurgiu o interesse no tratamento das lesões bifurcadas, com a criação de novas técnicas complexas (dois stents) e stents dedicados para esse subgrupo ${ }^{4-6}$. Entretanto, a melhor estratégia de tratamento a ser empregada (um stent vs. dois stents) ainda não foi estabelecida de forma conclusiva. Além disso, questões relacionadas à trombose do stent permanecem como limitação desse método $^{7}$. Por esses motivos, avaliamos os resultados imediatos e tardios de uma série de pacientes com lesões bifurcadas pertencentes à prática clínica diária tratados exclusivamente com stents farmacológicos.

\section{MÉTODO}

\section{População}

Foram identificados todos os pacientes consecutivos tratados com implante de stents farmacológicos para lesões envolvendo pelo menos uma bifurcação coronária, no período de junho de 2002 a dezembro de $2006(n=156 / 1.036 ; 15 \%)$. Para o propósito deste estudo, a amostra de pacientes foi dividida em dois grupos, de acordo com a estratégia adotada no procedimento: grupo 1, implante de stent no ramo principal e angioplastia convencional no ramo lateral (um stent); e grupo 2, implante de stents em ambos os ramos (dois stents). Os pacientes submetidos a an-
1.04-7.68; $\mathrm{p}=0.04)$. Stent thrombosis rates using one stent was $1.9 \%$ vs. $3.9 \%$ using two stents; $p=0.6$ ). Conclusions: Drug-eluting stenting for coronary bifurcation lesions has shown low rates of combined events during long-term follow-up. The use of the two-stent strategy was associated with a higher rate of MACE, although this is a more complex subgroup.

DESCRIPTORS: Drug-eluting stents. Angioplasty, transluminal, percutaneous coronary/methods. Treatment outcome. Coronary stenosis/therapy.

gioplastia em vigência de síndromes coronarianas agudas, inclusive aqueles com o diagnóstico de infarto agudo do miocárdio com elevação do segmento ST, foram incluídos neste estudo. O critério de exclusão foi a presença de choque cardiogênico. Os dados relativos ao seguimento clínico foram obtidos de forma retrospectiva, por contato telefônico ou eletrônico (e-mail), e analisados quanto às taxas de ECAM.

\section{Definições}

Foram consideradas lesões em bifurcação as lesões coronarianas maiores que $50 \%$ envolvendo o ramo principal e/ou o ramo lateral (com diâmetro $\geq 2,0 \mathrm{~mm}$ ). Utilizou-se a classificação proposta por Medina et al. ${ }^{8}$ para a classificação das bifurcações segundo a distribuição da placa. O sucesso do procedimento foi definido como lesão residual $<20 \%$ no vaso principal e $<50 \%$ no ramo lateral, por estimativa visual, com fluxo distal normal (TIMI 3). Os ECAM incluíram óbito por qualquer causa, infarto ou necessidade de nova RVA. Considerou-se RVA qualquer nova intervenção no vaso principal ou no ramo lateral. Definiu-se como revascularização da lesão-alvo (RLA) qualquer reintervenção no segmento tratado, que incluía os $5 \mathrm{~mm}$ proximais e distais à lesão. As taxas de RVA e de RLA foram calculadas cumulativamente para ramos principal e lateral. Infartos periprocedimento foram considerados se observada elevação enzimática (CKMB) superior a três vezes o limite superior do valor considerado normal para o método, mesmo na ausência de sintomas.

Os eventos foram computados de forma hierárquica. Avaliou-se a taxa de trombose do stent segundo os critérios estabelecidos pelo Academic Research Consortium $^{9}$, que define a ocorrência como definitiva, provável ou possível e, temporalmente, como aguda (ocorrendo nas primeiras 24 horas), subaguda (ocorrendo entre 24 horas e 30 dias após o procedimento), tardia (entre 31 dias e um ano) e muito tardia (após um ano).

\section{Intervenção coronariana percutânea}

Todos os pacientes foram pré-tratados com ácido acetilsalicílico e clopidogrel na dose de 300 mg ou 
Almeida BO, et al. Resultados Imediatos e Tardios do Implante de Stents Farmacológicos para o Tratamento de Bifurcações: Comparação entre as Estratégias de Um Stent Versus Dois Stents. Rev Bras Cardiol Invas. 2008;16(4):415-421.

$600 \mathrm{mg}$. Durante o procedimento, os pacientes receberam heparina não-fracionada na dose de $100 \mathrm{UI} / \mathrm{kg}$ ou $70 \mathrm{UI} / \mathrm{kg}$ (associada a abciximab) ajustada de modo a obter-se o tempo de coagulação ativada, mensurado pelo equipamento Hemocron 801, entre 300 e 350 segundos ou 250 a 300 segundos (com o uso de abciximab). A decisão pela administração de abciximab esteve a critério do operador responsável pela intervenção. A duração do regime de antiagregação dupla foi definida pelo cardiologista clínico responsável pelo caso, obedecendo às recomendações mínimas vigentes no momento do implante.

Coube unicamente ao intervencionista a decisão pela estratégia de tratamento adotada (um stent vs. dois stents). A escolha entre as diversas técnicas que utilizam dois stents ( $\mathrm{V}$-stent, simultaneous kissing stent, crush, T-stent ou cullote), assim como o stent farmacológico implantado, foi feita exclusivamente de acordo com o julgamento individualizado do operador e com a disponibilidade comercial do dispositivo (Cypher: a partir de maio de 2002; Taxus: a partir de maio de 2003; e CoStar: a partir de abril de 2006). Os procedimentos foram realizados de acordo com as técnicas previamente descritas e formalizadas nas diretrizes de intervenção coronariana vigentes.

\section{Análise estatística}

As variáveis categóricas estão representadas pelo número absoluto ou porcentual (\%) e foram comparadas pelo teste do qui-quadrado ou teste exato de Fisher. As variáveis numéricas estão representadas pela média e desvio padrão ou mediana com variação interquartil e foram comparadas pelo teste $t$ de Student. As curvas de sobrevida livre de eventos adversos foram avaliadas pelo método de Kaplan-Meier e comparadas pelo teste de log-rank. A análise univariada foi realizada para identificar os fatores relacionados à ocorrência de ECAM no seguimento clínico tardio. Realizou-se, ainda, análise multivariada para identificar os fatores preditores independentes de ECAM, utilizando-se nessa análise todas as variáveis com nível de significância $<0,2$ obtidos na análise univariada. Os resultados estão representados por odds ratio $(\mathrm{OR})$ e seus respectivos intervalos de confiança de 95\% (IC 95\%). Considerou-se indicativo de significância estatística a presença de $\mathrm{p}<0,05$.

A análise estatística foi realizada com auxílio do programa estatístico SPSS Inc., versão 13.0 (Chicago, Illinois, Estados Unidos).

\section{RESULTADOS}

No total, a média das idades foi de $64 \pm 12$ anos, $75,6 \%$ dos pacientes eram do sexo masculino e $28,8 \%$ eram diabéticos. O grupo 1 (um stent) compreendia $105(67,3 \%)$ pacientes e o grupo 2 (dois stents) foi composto por $51(32,7 \%)$ pacientes. As características clínicas e demográficas basais estão apresentadas na
Tabela 1. O grupo 1 foi composto por indivíduos com maior incidência de hipertensão (63,8\% vs. 49,0\%; $p=0,03)$ e dislipidemia $(49,5 \%$ vs. $35,3 \% ; p=0,04)$. Não houve diferenças entre as demais variáveis. A Tabela 2 sumariza as características angiográficas e do procedimento. Observa-se que, no grupo 1, o diâmetro de referência e o diâmetro de estenose do ramo lateral foram significativamente menores que no grupo $2(2,44 \mathrm{~mm} \pm 0,41 \mathrm{~mm}$ vs. $2,71 \mathrm{~mm} \pm 0,33 \mathrm{~mm}$, $p<0,01$ e $54 \% \pm 32 \%$ vs. $79 \% \pm 19 \%$, p < 0,01, respectivamente).

Observa-se, também, que as lesões envolvendo ambos os ramos (bifurcações verdadeiras) ocorreram mais freqüentemente no grupo 2 em comparação ao grupo 1 (84,3\% vs. 55,2\%; p < 0,01). A distribuição das lesões, de acordo com a classificação de Medina et al. ${ }^{8}$, está apresentada na Figura 1.

TABELA 1

Características demográficas e clínicas basais

\begin{tabular}{lccr}
\hline & $\begin{array}{c}\text { 1 stent } \\
(\mathbf{n}=\mathbf{1 0 5})\end{array}$ & $\begin{array}{c}\text { 2 stents } \\
(\mathbf{n}=\mathbf{5 1})\end{array}$ & p \\
\hline Idade, anos & $64 \pm 12$ & $66 \pm 13$ & 0,22 \\
Masculino (\%) & $79(75,2)$ & $39(76,5)$ & $>0,99$ \\
HAS (\%) & $67(63,8)$ & $25(49,0)$ & 0,03 \\
Diabetes (\%) & $30(28,6)$ & $15(29,4)$ & 0,85 \\
Dislipidemia (\%) & $52(49,5)$ & $18(35,3)$ & 0,04 \\
Tabagismo atual (\%) & $24(22,8)$ & $13(25,5)$ & 0,78 \\
Quadro clínico & & & 0,53 \\
Angina estável/ & & & \\
Isquemia silenciosa (\%) & $42(40,0)$ & $17(33,4)$ & \\
IAM sem supra ST (\%) & $19(18,1)$ & $10(19,6)$ & \\
IAM com supra ST (\%) & $7(6,7)$ & $4(7,8)$ &
\end{tabular}

Valores = média \pm 1 desvio padrão ou pela freqüência $\mathrm{n}(\%)$. HAS = hipertensão arterial sistêmica; IAM = infarto agudo do miocárdio; $\mathrm{n}=$ número de pacientes.

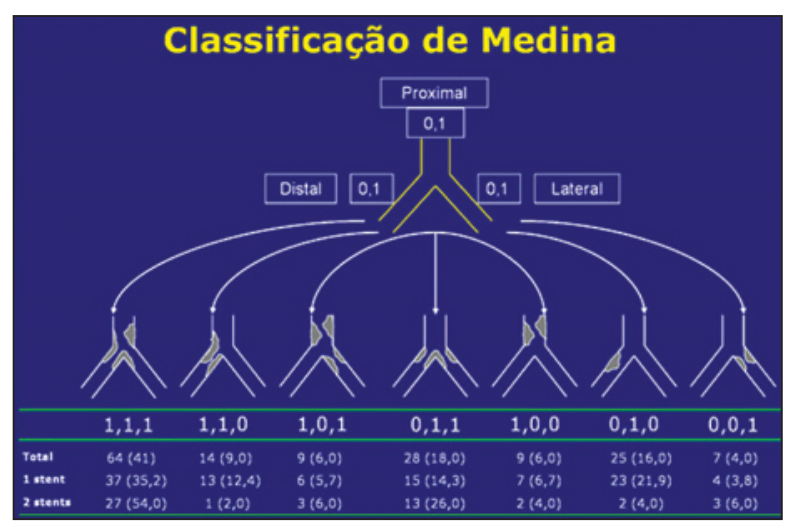

Figura 1 - Distribuição das lesões de acordo com a classificação de Medina et al. ${ }^{8}$. 
Quando utilizado, o implante de dois stents foi realizado pela técnica de crush em $23(14,7 \%)$ casos, T-stent em $16(10,3 \%)$, cullote em $5(3,2 \%)$, simultaneous kissing stent em 6 (3,8\%), e V-stent em $1(0,6 \%)$ caso. Notam-se diferenças entre os grupos no que se refere à realização de angioplastia por técnica de kissing balloon no final do procedimento. No grupo 1, 94,3\% dos pacientes foram submetidos a kissing balloon vs. 68,6\% no grupo 2 ( $p<0,01)$. O índice de insucesso angiográfico no ramo lateral foi observado em maior proporção no grupo $1(15,2 \%$ vs. 3,9\%; p = 0,05) (Tabela 2).

\section{Seguimento clínico}

Foram obtidas informações referentes ao seguimento clínico de 151 (96,8\%) pacientes, que, na média, foi de 2,4 $\pm 1,3$ anos. Os eventos foram analisados e adjudicados por dois cardiologistas intervencionistas e estão demonstrados, de forma cumulativa, na Tabela 3. A incidência de ECAM foi de 9,5\% no grupo 1 vs. $21,6 \%$ no grupo 2 ( $p=0,05)$, à custa de maior necessidade de RLA $(11,8$ vs. 1,9; $p=0,01)$ e de RVA $(13,7 \%$ vs. $4,8 \% ; p=0,06)$ no grupo 2 vs. grupo 1 , respectivamente, como demonstrado na Figura 2. Não houve diferenças significativas entre os grupos em relação à incidência de óbito $(4,8 \%$ vs. 9,8\%; $p=0,30)$ ou infarto $(2,9 \%$ vs. $5,9 \% ; p=0,39)$.

Não foi observada diferença estatisticamente significativa em relação à incidência de trombose (definitiva ou provável) entre os grupos, sendo demonstrados dois casos em cada grupo $(1,9 \%$ vs. $3,9 \% ; p=0,60)$. As curvas de sobrevida livre de ECAM estão apresentadas na Figura 3.

Na análise univariada, representada na Tabela 4, foram testadas as características clínicas, angiográficas e do procedimento. As variáveis que atingiram nível de significância preestabelecido (técnica com dois stents, kissing balloon final, diâmetro de referência pré-procedimento do ramo lateral) foram avaliadas no modelo multivariado. O tratamento de lesões em bifurcações verdadeiras não foi preditor de eventos. Na análise multivariada (Tabela 5), identificou-se exclusivamente a utilização de dois stents como preditor independente de ECAM (OR: 2,82; IC 95\%: 1,04-7,68; $p=0,04$ ).

\section{DISCUSSÃO}

\section{Resultados cumulativos}

Os principais achados da nossa investigação são: 1) os seguimentos tardio e muito tardio de lesões

TABELA 3

Eventos clínicos acumulados

\begin{tabular}{lccc}
\hline & $\begin{array}{c}\text { 1 stent } \\
(\mathbf{n}=\mathbf{1 0 5})\end{array}$ & $\begin{array}{c}\text { 2 stents } \\
(\mathbf{n}=\mathbf{5 1})\end{array}$ & $\mathbf{p}$ \\
\hline ECAM (\%) & $10(9,5)$ & $11(21,6)$ & 0,05 \\
Óbito (\%) & $5(4,8)$ & $5(9,8)$ & 0,30 \\
Infarto (\%) & $3(2,9)$ & $3(5,9)$ & 0,39 \\
RLA (VP ou RL) (\%) & $2(1,9)$ & $6(11,8)$ & 0,01 \\
RVA (VP ou RL) (\%) & $5(4,8)$ & $7(13,7)$ & 0,06 \\
Trombose definitiva & & & \\
ou provável (\%) & $2(1,9)$ & $2(3,9)$ & 0,60 \\
\hline
\end{tabular}

ECAM = eventos cardíacos adversos maiores; $\mathrm{n}=$ número de pacientes; $\mathrm{RL}=$ ramo lateral; $\mathrm{RLA}=$ revascularização da lesão-alvo; RVA = revascularização do vaso-alvo; VP = vaso principal.

TABELA 2

Características angiográficas e do procedimento

\begin{tabular}{lccc}
\hline & 1 stent $(\mathbf{n}=\mathbf{1 0 5})$ & $\mathbf{2}$ stents $(\mathbf{n}=\mathbf{5 1})$ & $\mathbf{p}$ \\
\hline Abciximab (\%) & $30(28,6)$ & $19(37,3)$ & 0,23 \\
Bifurcação verdadeira (\%) & $58(55,2)$ & $43(84,3)$ & $<0,01$ \\
Diâmetro de referência VP (mm) & $3,19 \pm 0,33$ & $3,21 \pm 0,32$ & 0,79 \\
Diâmetro de referência RL (mm) & $2,44 \pm 0,41$ & $2,71 \pm 0,33$ & $<0,01$ \\
Diâmetro de estenose pré VP (\%) & $84 \pm 14$ & $83 \pm 17$ & 0,84 \\
Diâmetro de estenose pré RL (\%) & $54 \pm 32$ & $79 \pm 19$ & $<0,01$ \\
Diâmetro de estenose pós VP (\%) & $10 \pm 3,8$ & $11 \pm 6,5$ & 0,28 \\
Diâmetro de estenose pós RL (\%) & $27 \pm 20,5$ & $13 \pm 9,5$ & $<0,01$ \\
Comp. stent VP (mm) & $28 \pm 18$ & $25 \pm 13$ & 0,43 \\
Kissing balloon final (\%) & $99(94,3)$ & $35(68,6)$ & $2(3,9)$ \\
Insucesso RL (\%) & $16(15,2)$ & $1(2,0)$ & 0,01 \\
Insucesso VP (\%) & $0(0,0)$ & 0,05 \\
& & 0,32 \\
\hline
\end{tabular}

Valores $=$ média \pm 1 desvio padrão ou pela freqüência $\mathrm{n}(\%)$. Análise angiográfica visual. Comp. $=$ comprimento; $\mathrm{n}=$ número de pacientes; $\mathrm{RL}=$ ramo lateral; $\mathrm{VP}=$ vaso principal. 


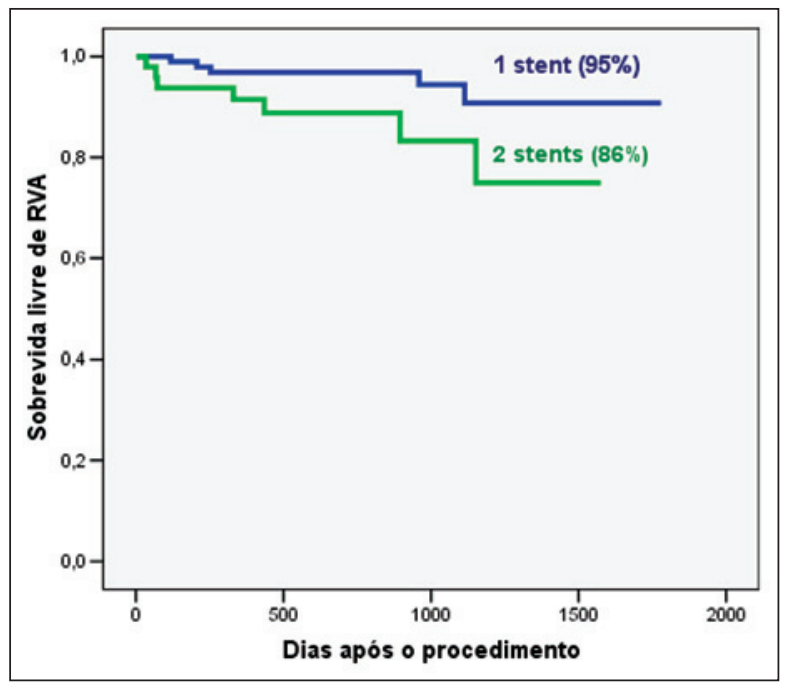

Figura 2 - Curvas de Kaplan-Meier da sobrevida livre de revascularização do vaso-alvo (vaso principal e ramo lateral) (teste do log-rank, $\mathrm{p}=0,031)$. RVA = revascularização do vaso-alvo.

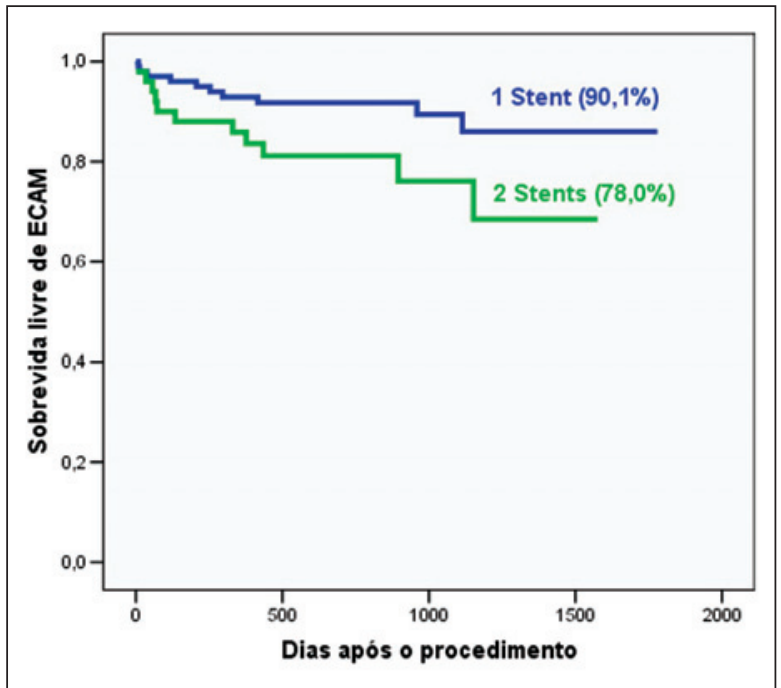

Figura 3 - Curvas de Kaplan-Meier da sobrevida livre de eventos cardíacos adversos maiores (teste do log-rank, $\mathrm{p}=0$ 0,033). ECAM = eventos cardíacos adversos maiores.

bifurcadas tratadas no universo dos stents farmacológicos apresentaram baixa taxa de ECAM; 2) observaram-se taxas de necessidade de nova RVA por reestenose clínica (vaso principal ou ramo lateral) de 7,7\% (12 pacientes); 3) o insucesso do ramo lateral não se correlacionou com a incidência de ECAM; 4) o grupo tratado por estratégia complexa (dois stents) apresentou maior número de ECAM, mesmo após ajuste para as características clínicas e angiográficas.

No presente estudo, demonstramos resultados imediatos e tardios encorajadores no tratamento de lesões bifurcadas no universo dos stents farmacológicos, com sobrevida livre de ECAM de $86,6 \%$ no seguimento clínico médio de 2,4 anos. Além do seguimento clínico mais prolongado em comparação com os registros e estudos randomizados publicados nesse contexto, esses resultados encontram-se de acordo com o subestudo do ARTS II-bifurcação, em que a taxa de ECAM foi de 13,3\% no seguimento de um ano $^{10}$. Esses resultados refletem melhora no tratamento das lesões bifurcadas em comparação com o controle histórico da era dos stents convencionais, em que as taxas de eventos se situavam em torno de $32 \%$, com taxas de nova intervenção percutânea em torno de $16,9 \%$ e necessidade de cirurgia de revascularização miocárdica em aproximadamente $10 \%$ dos pacientes ${ }^{11}$. Em nosso estudo, observamos incidência de nova intervenção de $7,7 \%$, não tendo sido necessária revascularização cirúrgica em nenhum dos pacientes. Isso reflete a natureza predominantemente focal e usualmente de fácil abordagem percutânea das reestenoses clínicas após implante de stent farmacológicos ${ }^{12}$, e encontra-se em conformidade com o registro ARTS II, em que a taxa de revascularização cirúrgica foi de $1,5 \%{ }^{10}$.

\section{Trombose dos stents}

O tratamento de lesões bifurcadas é conhecido como preditor independente de trombose dos stents ${ }^{7,13,14}$. Estudos demonstram taxas de trombose do stent que variam entre $1,5 \%$ e $4,3 \%$ na dependência da definição da trombose do stent (protocolar vs. critérios ARC $)^{10,15-17}$. Mais ainda, quando se avaliam as bifurcações tratadas com implante de dois stents farmacológicos, essas taxas podem atingir valores acima de $6 \%{ }^{18}$. No nosso estudo detectamos taxa global de trombose de 2,5\%, similar à reportada na literatura. De fato, os pacientes tratados com dois stents apresentaram incidência de trombose de 3,9\%, que não diferiu estatisticamente do grupo com um stent $(p=0,6)$. Esses dados poderiam ser explicados pela complexidade técnica do procedimento e a sobreposição de hastes metálicas, polímeros e fármacos na $\operatorname{carina}^{19}$.

\section{Estratégia de um stent vs. dois stents}

$\mathrm{Na}$ análise multivariada, demonstrou-se que a técnica com dois stents foi a única preditora independente para os ECAM (OR: 2,82; IC 95\%: 1,04$7,68 ; p=0,04)$. Isso demonstra que, a despeito do melhor desempenho dos stents farmacológicos em comparação com o controle histórico dos stents convencionais, a estratégia com o implante de dois stents permanece associada a maior probabilidade de $\mathrm{ECAM}^{2,18}$. Paralelamente, observamos que, apesar da taxa de insucesso do ramo lateral pela definição angiográfica na estratégia com um stent, esse achado não determinou maior chance de eventos. De fato, publicações relacionadas à avaliação fisiológica da estenose residual do ramo lateral após implante de stent no ramo principal demonstram que o grau de 
TABELA 4

Análise univariada para eventos cardíacos adversos maiores

\begin{tabular}{lccccc}
\hline & $\boldsymbol{\beta}$ & Erro padrão & OR & $\mathbf{p}$ & IC $\mathbf{9 5} \%$ \\
\hline Dois stents vs. um stent & 0,98 & 0,47 & 2,63 & 0,04 & $1,03-6,71$ \\
Diabetes & $-0,24$ & 0,55 & 0,78 & 0,66 & $0,26-2,33$ \\
Bifurcação verdadeira & 0,40 & 0,47 & 1,04 & 0,93 & $0,41-2,64$ \\
Sexo & $-0,36$ & 0,59 & 0,69 & 0,53 & $0,21-2,20$ \\
Diâmetro de referência pré VP & 0,40 & 0,70 & 1,49 & 0,57 & $0,37-5,97$ \\
Diâmetro de referência pré RL & 0,70 & 0,54 & 2,02 & 0,19 & $0,69-5,90$ \\
Comp. stent VP & $-0,03$ & 0,15 & 0,99 & 0,83 & $0,96-1,02$ \\
Kissing final & $-0,86$ & 0,58 & 0,42 & 0,13 & $0,13-1,31$ \\
Insucesso RL & 0,32 & 0,68 & 1,38 & 0,63 & $0,36-5,28$
\end{tabular}

Comp. = comprimento; IC 95\% = intervalo de confiança de 95\%; OR = odds ratio; $\mathrm{RL}=$ ramo lateral; $\mathrm{VP}$ = vaso principal.

TABELA 5

Análise multivariada para eventos cardíacos adversos maiores

\begin{tabular}{lcccrr} 
& $\boldsymbol{\beta}$ & Erro padrão & OR & p & IC 95\% \\
\hline Dois stents vs. um stent & 1,04 & 0,51 & 2,82 & 0,04 & $1,04-7,68$ \\
Diabetes & $-0,14$ & 0,54 & 0,86 & 0,78 & $0,29-2,51$ \\
Diâmetro de referência pré RL & 0,40 & 0,55 & 2,02 & 0,19 & $0,69-5,90$ \\
Kissing final & 0,10 & 0,62 & 1,51 & 0,46 & $0,50-4,45$
\end{tabular}

IC $95 \%$ = intervalo de confiança de $95 \%$; OR = odds ratio; $\mathrm{RL}=$ ramo lateral.

estenose residual angiográfica do ramo lateral pode não ser funcionalmente significativo ${ }^{20}$.

\section{Limitações}

Este estudo carece das virtudes de um estudo randomizado e apresenta todas as limitações de um registro de seguimento exclusivamente clínico, refletindo as baixas taxas de reestenose nesse contexto. Mais ainda, é possível que a escolha da estratégia tenha sido influenciada, em parte, pela complexidade das lesões, determinando algum desequilíbrio entre os grupos.

\section{CONCLUSÕES}

Este estudo documenta os seguimentos tardio e muito tardio, pouco reportados anteriormente, de lesões bifurcadas tratadas exclusivamente com o implante de stents farmacológicos. Mais que uma comparação entre as técnicas empregadas (um stent vs. dois stents), este registro demonstra que o tratamento das bifurcações evoluiu após o advento dos stents farmacológicos. Acreditamos que o implante sistemático de dois stents deva ser evitado e reservado para casos de maior complexidade angiográfica. A despeito da maior gravidade, freqüentemente observada nos pacientes tratados com as técnicas complexas, fora dos estudos randomizados, esses dados apontam para meIhora progressiva nos resultados desse subgrupo com o uso dos stents farmacológicos.

\section{REFERÊNCIAS BIBLIOGRÁFICAS}

1. Garot $P$, Lefevre $T$, Savage $M$, Louvard $Y$, Bamlet $W R$, Willerson JT, et al. Nine-month outcome of patients treated by percutaneous coronary interventions for bifurcation lesions in the recent era: a report from the Prevention of Restenosis with Tranilast and its Outcomes (PRESTO) trial. J Am Coll Cardiol. 2005;46(4):606-12.

2. Al Suwaidi J, Berger PB, Rihal CS, Garratt KN, Bell MR, Ting $\mathrm{HH}$, et al. Immediate and long-term outcome of intracoronary stent implantation for true bifurcation lesions. J Am Coll Cardiol. 2000;35(4):929-36.

3. Yamashita T, Nishida T, Adamian MG, Briguori C, Vaghetti $\mathrm{M}$, Corvaja $\mathrm{N}$, et al. Bifurcation lesions: two stents versus one stent-immediate and follow-up results. J Am Coll Cardiol. 2000;35(5):1145-51.

4. Colombo A, Stankovic G, Orlic D, Corvaja N, Liistro F, Airoldi $F$, et al. Modified T-stenting technique with crushing for bifurcation lesions: immediate results and 30-day outcome. Catheter Cardiovasc Interv. 2003;60(2):145-51.

5. Schampaert E, Fort S, Adelman AG, Schwartz L. The Vstent: a novel technique for coronary bifurcation stenting. Cathet Cardiovasc Diagn. 1996;39(3):320-6.

6. Sharma SK. Simultaneous kissing drug-eluting stent technique for percutaneous treatment of bifurcation lesions in large-size vessels. Catheter Cardiovasc Interv. 2005;65(1):10-6. 
7. lakovou I, Schmidt T, Bonizzoni E, Ge L, Sangiorgi GM, Stankovic G, et al. Incidence, predictors, and outcome of thrombosis after successful implantation of drug-eluting stents. JAMA. 2005;293(17):2126-30.

8. Medina A, Suarez de Lezo J, Pan M. A new classification of coronary bifurcation lesions. Rev Esp Cardiol. 2006;59(2):183.

9. Cutlip DE, Windecker S, Mehran R, Boam A, Cohen DJ, van Es GA, et al. Clinical end points in coronary stent trials: a case for standardized definitions. Circulation. 2007;115(17): 2344-51.

10. Tsuchida K, Colombo A, Lefevre T, Oldroyd KG, Guetta V, Guagliumi G, et al. The clinical outcome of percutaneous treatment of bifurcation lesions in multivessel coronary artery disease with the sirolimus-eluting stent: insights from the Arterial Revascularization Therapies Study part II (ARTS II). Eur Heart J. 2007;28(4):433-42.

11. Al Suwaidi J, Yeh W, Cohen HA, Detre KM, Williams DO, Holmes DR Jr. Immediate and one-year outcome in patients with coronary bifurcation lesions in the modern era (NHLBI dynamic registry). Am J Cardiol. 2001;87(10):1139-44.

12. Park $\mathrm{CB}$, Hong $\mathrm{MK}, \mathrm{Kim} \mathrm{YH}$, Park DW, Han $\mathrm{KH}$, Lee CW, et al. Comparison of angiographic patterns of in-stent restenosis between sirolimus- and paclitaxel-eluting stent. Int J Cardiol. 2007;120(3):387-90.

13. Angiolillo DJ, Fernandez-Ortiz A, Bernardo E, Ramirez C, Barrera-Ramirez C, Sabate $M$, et al. Identification of low responders to a 300-mg clopidogrel loading dose in patients undergoing coronary stenting. Thromb Res. 2005; 115(1-2):101-8.
14. Kuchulakanti PK, Chu WW, Torguson R, Ohlmann P, Rha SW, Clavijo LC, et al. Correlates and long-term outcomes of angiographically proven stent thrombosis with sirolimus- and paclitaxel-eluting stents. Circulation. 2006;113(8):1108-13.

15. Hoye A, lakovou I, Ge L, van Mieghem CA, Ong AT, Cosgrave J, et al. Long-term outcomes after stenting of bifurcation lesions with the "crush" technique: predictors of an adverse outcome. J Am Coll Cardiol. 2006;47(10):1949-58.

16. Ge L, Airoldi F, lakovou I, Cosgrave J, Michev I, Sangiorgi GM, et al. Clinical and angiographic outcome after implantation of drug-eluting stents in bifurcation lesions with the crush stent technique: importance of final kissing balloon post-dilation. J Am Coll Cardiol. 2005;46(4):613-20.

17. Ge L, Tsagalou E, lakovou I, Sangiorgi GM, Corvaja N, Airoldi $F$, et al. In-hospital and nine-month outcome of treatment of coronary bifurcational lesions with sirolimus-eluting stent. Am J Cardiol. 2005;95(6):757-60.

18. Colombo A, Moses JW, Morice MC, Ludwig J, Holmes DR $\mathrm{Jr}$, Spanos V, et al. Randomized study to evaluate sirolimuseluting stents implanted at coronary bifurcation lesions. Circulation. 2004;109(10):1244-9.

19. Virmani R, Guagliumi G, Farb A, Musumeci G, Grieco N, Motta $\mathrm{T}$, et al. Localized hypersensitivity and late coronary thrombosis secondary to a sirolimus-eluting stent: should we be cautious? Circulation. 2004;109(6):701-5.

20. Koo BK, Kang HJ, Youn TJ, Chae IH, Choi DJ, Kim HS, et al. Physiologic assessment of jailed side branch lesions using fractional flow reserve. J Am Coll Cardiol. 2005;46(4): 633-7. 